\title{
A Simple Approach for Controlling an Open-Source Syringe Pump
}

\author{
Fatih Akkoyun ${ }^{1}\left(\mathbb{D}\right.$, Adem Ozcelik $^{1 *}$ (1) \\ 'Aydın Adnan Menderes University, Department of Mechanical Engineering, Turkey
}

\begin{abstract}
Precise control of fluid flows in microfluidics is crucial for various applications in lab-on-a-chip and pointof-care diagnostics. Standard bench-top equipment for providing this capability are syringe pumps. However, high cost of these systems limit their availability in low resourced laboratories. There are various open-sourced alternative syringe pump systems that can be fabricated and assembled using 3D printing, but they lack versatile control and flow rate characterization that are required for microfluidic applications. We report a simple and cost-effective approach to control an open-source multi-channel syringe pump. Simultaneous and adjustable flow control, and detailed characterization of the volume flow rates for different syringe volumes are also demonstrated.
\end{abstract}

Keywords: syringe pump, microcontroller, microfluidics, flow control, flow pro

\section{INTORDUCTION}

Microfluidics is a growing field with promising potential in lab-on-a-chip and point-of-care diagnostics that enables various advantages such as precision and reduced contamination in chemical, biological, medical and engineering fields [1-3]. Flow rate control in microfluidic systems is an important requirement for chemical reactions, nanoparticle synthesis, bioanalysis, and therapeutic applications [4-7]. Syringe pumps are the benchtop equipment for regulating fluid flow in microfluidics, but they are not easily accessible to limited resource laboratories due their relatively high costs [8]. Therefore, there is a need for a low-cost, easy-to-fabricate, and practical syringe pump for enabling versatile and high precision fluid flow control in the domain of microfluidic research.

There have been several reports on various open-source laboratory equipment including syringe pumps $[9,10]$. In one of the earliest demonstrations, Wijnen et al. designed and fabricated an open-source syringe pump using CAD and 3D printing [8]. A Raspberry Pi computer was used to control a stepper motor through a Pololu A4988 stepper motor controller. Linear motion for the syringe infusion and withdraw was obtained by coupling an M5 threaded rod to the stepper motor and moving a carriage with an embedded M5 nut. A web paged-based graphical user interface (GUI) was designed to control the system. Flow rate characterization and accuracy were not discussed in this study. In a different work,
Juarez et al. presented a low-cost syringe pump targeted intravenous infusions in hospitals [11]. The working principle of this pump was based on the controlled-release of a loaded spring mechanism through a stepper motor which was driven by a microcontroller. Effectiveness of this syringe pump system was later tested via intravenously delivering magnesium sulfate to patients with pre-eclamtic condition [12]. In total, 22 women patients were included in a 466-hour long testing period, and lower than $2 \%$ errors were recorded in the applied flow rates. The reported achievable flow rates for this system were between 3 and $60 \mathrm{~mL} /$ hour which is relatively higher for microfluidic applications considering the lower flow rate requirements $[13,14]$. In a different study, Lake at al. developed a PID closed-loop feedback controlled syringe pump for microfluidic applications [15]. In this work, piezoresistive flow sensors were used to measure and correct for deviations from the flow rate set-points so that the intended and actual flow rates were matched with better precision. Arduino microcontroller boards and a stepper motor driver were employed to control the syringe infusion within 1\% error with the PID control method. Even though this work demonstrated a higher precision, the required flow sensor added further complexity and cost to the overall system that adversely affect the targeted low-cost goal. Guelig et al. presented a pressure driven syringe pump that did not require electricity or electronic control units to operate [16]. A pressure regulating valve and constant source of pressurized air were used to move the syringe plunger. Overall, this 
system provided flow rates from 0.5 to $8 \mathrm{~mL} / \mathrm{h}$ with less than $10 \%$ deviation from the desired flow rates. While this system provided operation without electricity and control electronics, the whole system became more complex with the pressure regulation and pressurize air requirements. Similar to the aforementioned systems, there have been recent examples of open-source and mostly 3D printed syringe pumps, but these systems either have an unreliable, limited and application specific GUI or their achievable flow rates were not tested for the values that are relevant for microfluidic applications [17-20]. For example, Garcia et al. developed a Raspberry Pi driven syringe pump that was also controlled through a touchscreen [21], but the GUI of the system did not allow a traditional independent flow rate control for the syringe infusion in the dual syringe pump. Furthermore, the specific stepper driver and touchscreen rendered the system slightly more expensive for low-budget laboratories. Therefore, there is still a need for a simpler, robust, and easy-to-use syringe pump that can consistently output a range of flow rates for microfluidic applications.

Herein, we present a simple and reliable control of an open-source multi-channel syringe pump using an ATmega32A microcontroller and commonly available A4988 stepper drivers. The microcontroller and the GUI are coded using $\mathrm{C}$ and $\mathrm{C \#}$ programming languages, respectively. Flow rate modulation is performed by using the duty cycle tuning. The multi-channel syringe pump is tested and characterized using $3 \mathrm{~mL}, 5 \mathrm{~mL}$ and $10 \mathrm{~mL}$ syringes for various flow rates ranging from $0.5 \mu \mathrm{L} / \mathrm{s}$ to $17 \mu \mathrm{L} / \mathrm{s}$. With its independent and simultaneous operation capabilities, this syringe pump system can be used in numerous microfluidic applications from nanoparticle synthesis to bioanalysis and droplet generation to cell separation.

\section{MATERIALS AND METHODS}

The multi-channel syringe pump system is composed of a computer, a microcontroller, a communication unit, step drivers, step motors and a mechanic assembly to hold syringes. The details of the mechanical assembly can be found in similar open-source syringe pump systems in the literature [18]. The main components of the developed syringe pump control system are shown in Figure 1.

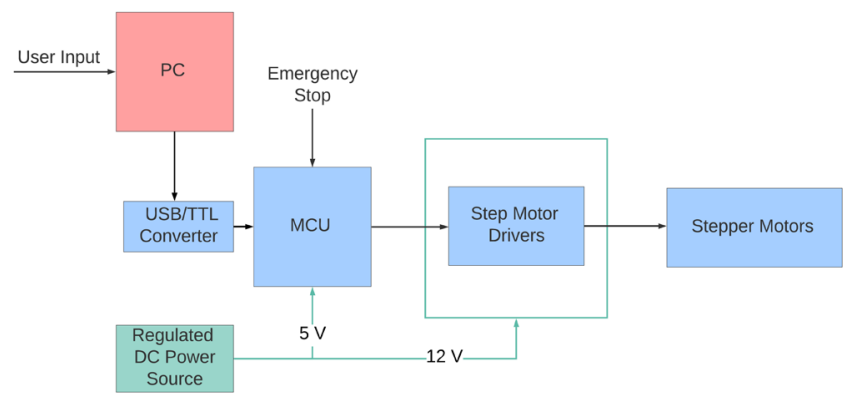

Figure 1. Main components and work-flow of the multi-channel syringe pump system.

A feedforward control system is designed to control the syringe pump considering the multi-channel flow. In this de- sign, three step motors are controlled via a computer-based software by determining the control parameters of the step motors with respect to the user inputs such as flow rate and syringe barrel diameter.

To compose the designed system, a graphical user interface (GUI) is developed to acquire inputs via a personal computer (PC). The GUI software is written in C\# language. Flow rates of the syringes are accepted as input from the user, and duty cycles with barrel diameters of the syringes are used to calculate the pulse counts for each step motor with respect to input parameters. Step motor parameters including step count, motor direction and duty cycle are transferred from the PC to the electronic control unit. The electronic control unit is composed of a microcontroller unit (MCU), a 16 $\mathrm{MHz}$ crystal and general-purpose input-output terminals (GPIO). The MCU is programmed using embedded $\mathrm{C}$ language. The task of the board is to receive, process and transmit the motor driving parameters from the PC to the step motor drivers. These parameters include motor ID, directions of the motors and step pulses corresponding to the duty cycles. The parameters are transmitted in a continuous loop from MCU to the step motor drivers to drive the step motors continuously. An external interrupt command is used to stop motors in an emergency, and an alert is shown to the user by the GUI. Step motor drivers are supplied with $12 \mathrm{~V}$ 5A power supply to provide adequate power for driving the step motors. The data flow direction of the designed system is shown in Figure 2 from GUI to step motors.

The GUI software as shown in Figure 3 works in three stages. In the first stage, the connection is established between the PC and the MCU to communicate with the syringe pump control system. This communication is accomplished using the FTDI chip which provides data transfer among USB and RS232 in serial. In the second stage, the flow rate, barrel diameter and direction parameters are accepted from the user over the GUI. In the last stage, the commands that used to control syringe pumps are generated by the software according to the inputs, and are transferred via serial communication when the run button is activated.

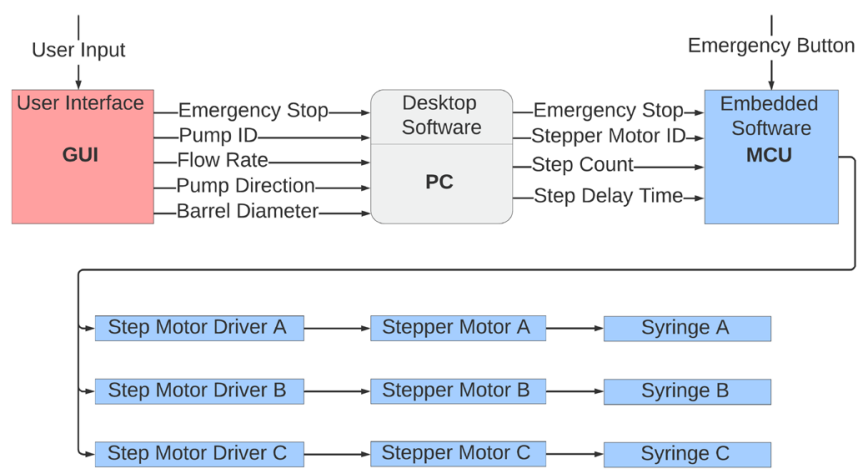

Figure 2. The data flow diagram of the system.

The syringe pump control commands generated by the software referencing the input parameters also can be transferred via the terminal screen manually. An example command is given as " $\$ \mathrm{STP}, 1, \mathrm{CCW}, 10,5$ " to run the Syringe 
Pump A for infusing a $5 \mathrm{ml}$ syringe with a $10 \mu \mathrm{l}$ flow rate. The control command is including a check string (\#STP), syringe pump selection (1-3), the direction of the syringe $(\mathrm{CW} / \mathrm{CCW})$, flow rate $(\mu \mathrm{l})$ and barrel diameter.

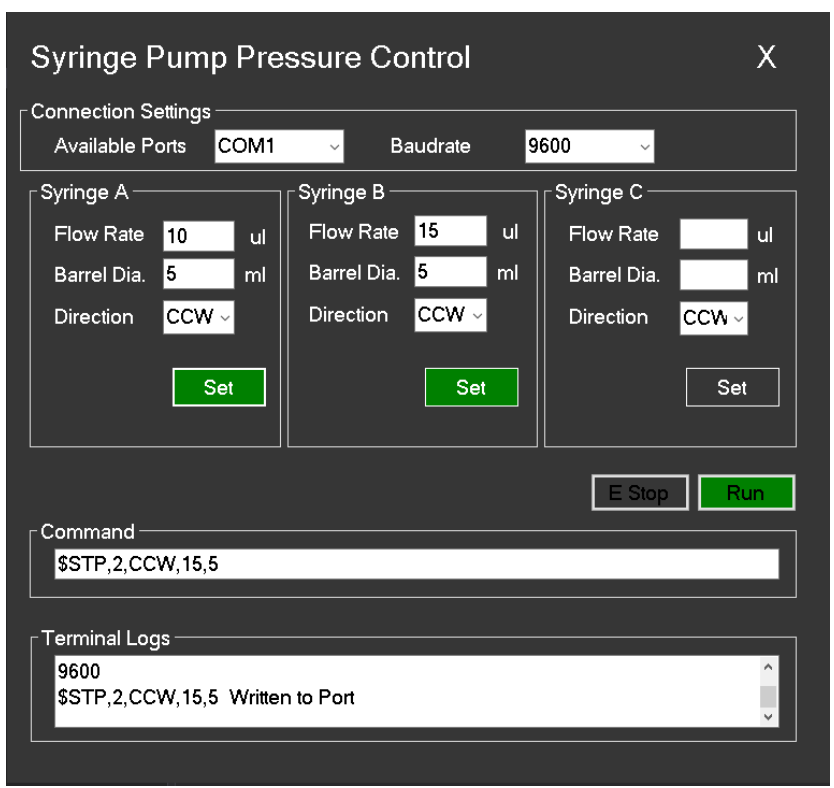

Figure 3. The GUI used to control the syringe pump system.

Embedded C language is used to program the MCU to receive, process and transfer the control commands. The obtained commands are parsed in this continuous program, and result parameters are assigned to each motor corresponding to the flow rate calculations. Syringe pump motor pulses and direction signals are transferred from MCU to the A4988 step motor drivers according to the input parameters within a loop. The connection drawing of the MCU, PC, step motor and drivers are shown in Figure 4. A $16 \mathrm{MHz}$ crystal and reset button connected, additionally.

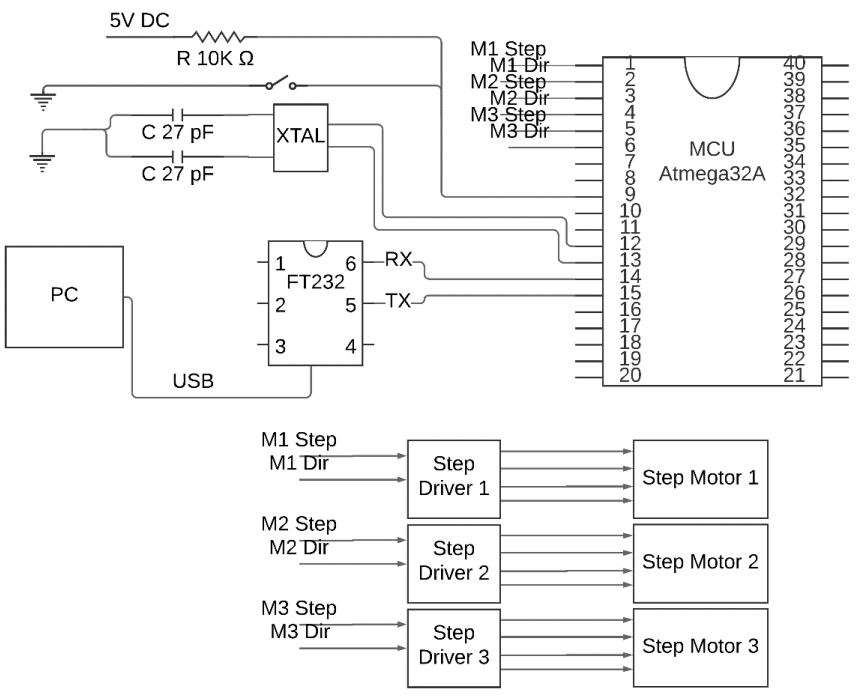

Figure 4. Wiring of the electronic components.

The mechanical part of the syringe pump is assembled using 3D printed PLA parts. Three sets of syringe holders were set side by side on a syringe base to hold and move syringes horizontally. Parts of the syringe pump system are printed using an Anycubic i3Mega printer. Later, the printed parts are assembled to form each pump unit. This approach enables a modular approach for the needs of different labs. The printed syringe holders are mounted on $5 \mathrm{~mm}$ threaded shafts and supported with linear bearings. NEMA 17 type step motor actuators are coupled with threaded shafts using couplers. The shafts are supported with double linear bearings for ensuring the smooth linear motion of the moving parts. The barrel flange is fixed on the base of the syringe pump and syringe barrels are tightened via perpendicularly positioned screws. To prevent the sliding of syringes during operation, plunger flanges are fitted in the slits. After fitting the barrels and plungers to the syringe pump mechanism, the actuators can move each plunger one by one respect to the user inputs via GUI of the system. Assembled image of the syringe pump mechanism is shown in Figure 5.

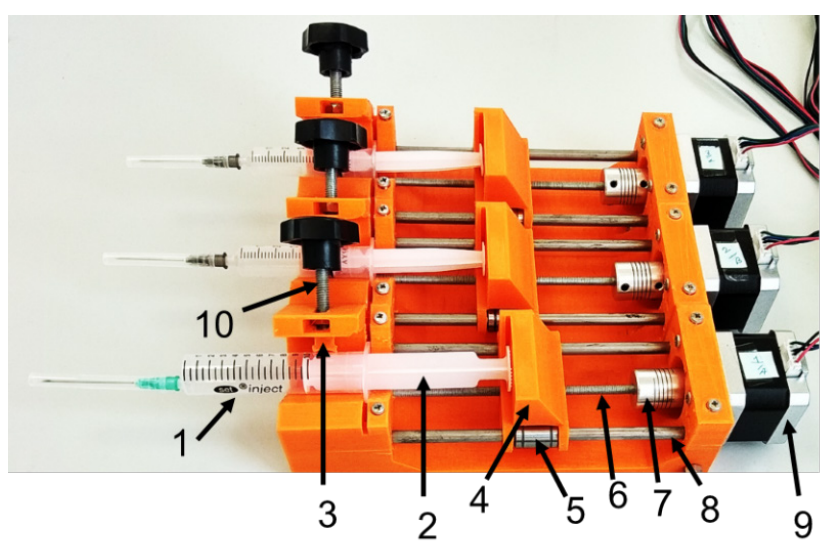

Figure 5. The frame of the syringe pump mechanism and assembly. 1: syringe barrel, 2: syringe plunger, 3: syringe holder, 4: plunger carrier, 5: linear ball bearing, 6: M5 threaded shaft, 7: shaft coupler, 8: $6 \mathrm{~mm}$ steel rod, 9: stepper motor, 10: syringe holder adjustment nob.

Experimental fluid flow profile observations are performed using an inverted Euromex microscope (OX.2053-PLPH, Euromex, Arnhem, Netherlands) equipped with Euromex HD colored camera (HD-Ultra, Euromex, Arnhem, Netherlands). Flow rate measurement videos are recorded using an entry level slow motion camera (Exilim EX-FC100, Casio, Tokyo, Japan).

\section{RESULTS AND DISCUSSION}

The flow rate output of the multi-channel syringe pump is characterized by using 3, 5, and $10 \mathrm{~mL}$ syringes. Experimental measurements of the flow rates for the different syringes are executed by measuring the distance and the time taken by the leading edge of the fluid in a polyethylene tube with an inner diameter of $0.59 \mathrm{~mm}$. For example, flow rate measurements of two $10 \mathrm{~mL}$ syringes for $5 \%$ duty cycle are shown in Figure 6. Different color food dyes are mixed with water to clearly mark the leading edge of the fluid in the tube. After each measurement, the tubes are flushed with water to clearly see the border of the fluid for the next measurement. From the measured distance and time, the speed of the flow is calculated. Then, the inner cross-sectional area of the tube is multiplied with the measured speed to obtain the volume flow rate using the following equation: 
$0 \mathrm{~s}$

2345678910111213141516171819202122232425

$0.7 \mathrm{~s}$

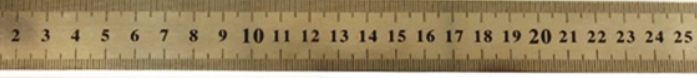

$1.4 \mathrm{~s}$

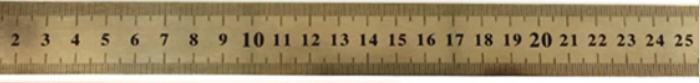

$2.1 \mathrm{~s}$

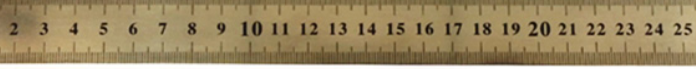

$2.8 \mathrm{~s}$

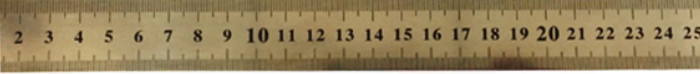

$3.5 \mathrm{~s}$

2345678 \& 10111213141516171819202122232425

Figure 6. Demonstration of volume flow rate measurements using red and blue colored water in a polyethylene tube. Time steps are measured from the individual frames of the experimental videos.

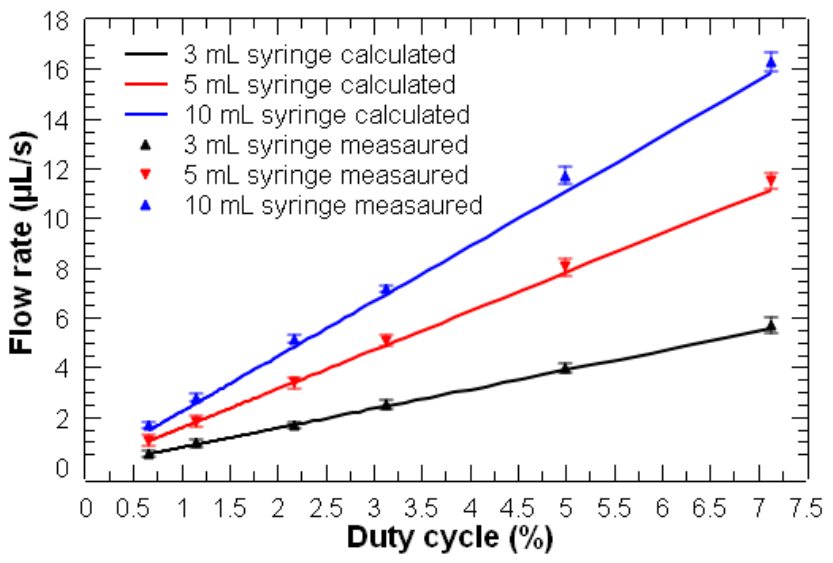

Figure 7. Experimental and calculated volume flow rates for 3, 5, and 10 $\mathrm{mL}$ syringes. Error bars are standard deviation of at least three different measurements for each data point.

Here, $\Delta \mathrm{x}, \Delta \mathrm{t}$, and $r$ are the distance travelled by the fluid, travel time, and the inner radius of the tube, respectively. The unit of the results of the equation 1 is converted to $\mu \mathrm{L} / \mathrm{s}$ for better representation of the flow rates for the microfluidic applications. For comparison to the experimental flow rates, the flow rate output of the system is also calculated using the time taken for one step of the stepper motors. This time step (1.01 x 10-4 seconds) and the pitch of the M5 threaded rod $(0.8 \mathrm{~mm})$ is used to calculate the speed of the plunger carrier. Using the inner cross-sectional area of each syringe type, flow rates for different syringes are calculated. Measured and calculated flow rates for the three types of the syringes are given in Figure 7. As seen from the figure, both measured and calculated flow rates are in good agreement. Error bars in the measured flow rates are the standard deviations of at least three different measurements for each data point. In Figure 7, three syringe types with 3, 5, and $10 \mathrm{~mL}$ volumes are used for the flow rate calculations and experiments because of their availability in our lab. Nevertheless, $1 \mathrm{~mL}$ syringes are also commonly used in biomedical appli- cations. Therefore, we have calculated the range of volumetric flow rates for a $1 \mathrm{~mL}$ syringe with an inner radius of 2.39 $\mathrm{mm}$. The linear displacement of the syringe plunger in this system depends on the pitch of the threaded shaft and also the duty cycle. Using the same pitch and duty cycle values used for the calculated flow rates shown in Figure7, the flow rates achievable with the $1 \mathrm{ml}$ syringe are found as $0.08 \mu \mathrm{L} / \mathrm{s}$, $0.28 \mu \mathrm{L} / \mathrm{s}, 0.52 \mu \mathrm{L} / \mathrm{s}, 0.75 \mu \mathrm{L} / \mathrm{s}$, and $1.71 \mu \mathrm{L} / \mathrm{s}$.

Flow profile output of the syringe pump system is also tested using a two inlet microfluidic channel. In this test, one of the inlets of the channel (inlet 1 ) is connected to a syringe filled with red colored water, and is kept at $10 \mu \mathrm{L} / \mathrm{s}$. The other inlet (inlet 2) is connected to a syringe filled with blue colored water, and the flow rate for this syringe is varied from 10 $\mu \mathrm{L} / \mathrm{s}$ to $2.5 \mu \mathrm{L} / \mathrm{s}$ with $2.5 \mu \mathrm{L} / \mathrm{s}$ steps. For each flow profile, gray scale values are also plotted along the white dotted line shown in Figure 8a. When both inlets are injected with 10 $\mu \mathrm{L} / \mathrm{s}$, width of the red and blue streams becomes equal at $\sim 100 \mu \mathrm{m}$. If inlet 2 is decreased to $7.5 \mu \mathrm{L} / \mathrm{s}$, the width of the red stream increases to $114.3 \mu \mathrm{m}$ (Figure 8b). Similarly, when the inlet 2 is decreased to 5 and $2.5 \mu \mathrm{L} / \mathrm{s}$, the blue stream increases to 133.3 (Figure 8c) and $160 \mu \mathrm{m}$ (Figure $8 d$ ), respectively. This test shows that various stable flow profiles can be generated using the simultaneous control of the syringe pump system.
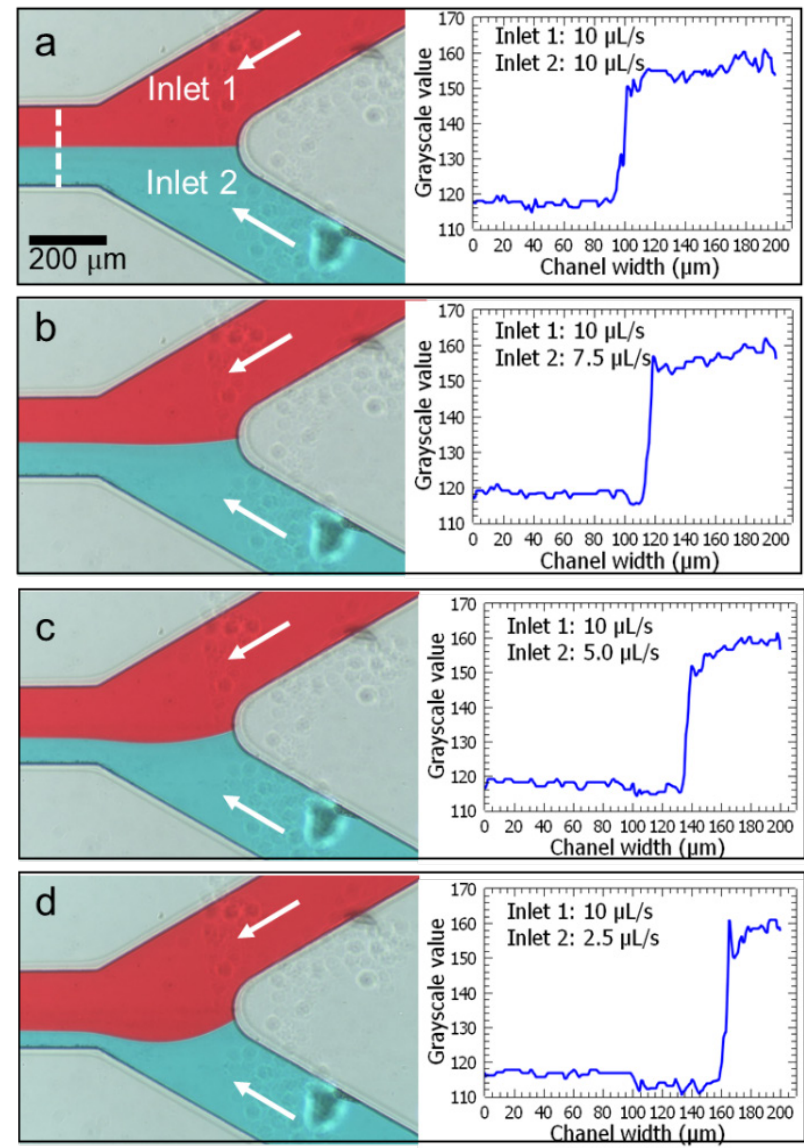

Figure 8. Fluid flow profiles are demonstrated in a 2-inlet microfluidic channel. a) When the inlet 1 and inlet two are both set to $10 \mu \mathrm{L} / \mathbf{s}$, stable and equal width flows are observed. As the flow rate of the inlet 2 is changed to b) $7.5 \mu \mathrm{L} / \mathbf{s}$, c) $5 \mu \mathrm{L} / \mathbf{s}$, and d) $2.5 \mu \mathrm{L} / \mathbf{s}$, the width of the blue colored flow infused from the inlet 2 decreases. Grayscale profiles plotted along the dashed line also indicates the shift of the laminar flow interface 
between two flows as the volume flow rate of the inlet 2 is decreased.

\section{CONCLUSION}

A robust and useful control of a multi-channel syringe pump system is demonstrated in this work. Using a microcontroller and functional GUI to control the syringe pump yields simultaneous or individual control of different size syringes. The characterization of the flow rates verifies the precision and example range of the volume flow rates for different syringe types. With the present system, flow rates from $0.1 \mu \mathrm{L} / \mathrm{s}$ to $18 \mu \mathrm{L} / \mathrm{s}$ can be achieved reliably. To obtain even lower flow rates, one can use a threaded shaft with a smaller pitch, smaller duty cycle, and stepper motors with smaller step sizes. These parameters control the linear displacement speed of the plunger carrier of the syringe pump. However, to decrease the pitch size, step size and the duty cycle, precision machined parts, more expensive stepper motors which are possible coupled with planet gears, and more capable motor controllers would be required. These improvements would increase the cost of the overall system. In this work, we target a low-budget system with good cost-performance balance. Simultaneous control of multiple flow lines provides parallel flow infusion which increases the workspace for the researchers. The present study focuses on the development of a multi-channel syringe pump for microfluidic applications based on a low-cost solution. The system demonstrated here can be a great asset for low budget laboratories to enable precise control of multiple flow lines for various microfluidic applications.

\section{5. REFERENCES}

[1] Weibel, D., Whitesides, G. (2006). Applications of microfluidics in chemical biology. Current Opinion in Chemical Biology, 10(6): 584-91. doi: 10.1016/j.cbpa.2006.10.016.

[2] Whitesides, G.M. (2006). The origins and the future of microfluidics. Nature, 442(7101): 368-73. doi: 10.1038/nature05058.

[3] Rivet, C., Lee, H., Hirsch, A., Hamilton, S., Lu, H. (2011). Microfluidics for medical diagnostics and biosensors. Chemical Engineering Science, 66(7): 1490-507. doi: 10.1016/j.ces.2010.08.015.

[4] Tosun, E., Ozgur, T., Ozgur, C., Ozcanli, M., Serin, H., Aydin, K. (2017). Comparative analysis of various modelling techniques for emission prediction of diesel engine fueled by diesel fuel with nanoparticle additives. European Mechanical Science, 1(1): 15-23. doi: 10.26701/ ems.320490.

[5] Kaleli, A.R. (2019). Gray based Fuzzy Gain-Scheduling PID Controller Design for Air-Fuel System Under Variable Engine Operating Conditions. European Mechanical Science, 3(4): 125-32. doi: 10.26701/ems.599452.

[6] Lu, M., Ozcelik, A., Grigsby, C.L.C.L. Zhao, Y., Guo, F., Leong, K.W.K.W., et al., (2016). Microfluidic hydrodynamic focusing for synthesis of nanomaterials. Nano Today, 11(6): 778-92. doi: 10.1016/j.nantod.2016.10.006.

[7] Li, Z., Mak, S.Y., Sauret, A., Shum, H.C. (2014). Syringe-pump-induced fluctuation in all-aqueous microfluidic system implications for flow rate accuracy. Lab on a Chip, 14(4): 744. doi: 10.1039/c3lc51176f.

[8] Wijnen, B., Hunt, E.J., Anzalone, G.C., Pearce, J.M. (2014). Open-Source Syringe Pump Library. PLoS ONE, 9(9): e107216. doi: 10.1371/ journal.pone.0107216.

[9] Pearce, J.M. (2014). Cut costs with open-source hardware. Nature, 505(7485): 618-618. doi: 10.1038/505618d.

[10] Pearce, J.M. (2012). Building Research Equipment with Free, Open-Source Hardware. Science, 337(6100): 1303-4. doi: 10.1126/ science. 1228183

[11] Juarez, A., Maynard, K., Skerrett, E., Molyneux, E., Richards-Kortum, R., Dube, Q., et al. (2016). AutoSyP: A Low-Cost, Low-Power Syringe Pump for Use in Low-Resource Settings. The American Journal of Tropical Medicine and Hygiene, 95(4): 964-9. doi: 10.4269/ajtmh.16-0285.

[12] Skerrett, E., Kommwa, E., Maynard, K., Juarez, A., Mataya, R., Richards-Kortum, R., et al. (2017). Evaluation of a low-cost, low-power syringe pump to deliver magnesium sulfate intravenously to pre-eclamptic women in a Malawian referral hospital. BMC Pregnancy and Childbirth, 17(1): 191. doi: 10.1186/s12884-017-1382-9.

[13] Zhang, P., Bachman, H., Ozcelik, A., Huang, T.J. (2020). Acoustic Microfluidics. Annual Review of Analytical Chemistry, 13(1): 17-43. doi: 10.1146/annurev-anchem-090919-102205.

[14] Wu, M., Ozcelik, A., Rufo, J., Wang, Z., Fang, R., Jun Huang, T. (2019). Acoustofluidic separation of cells and particles. Microsystems \& Nanoengineering, 5(1): 32. doi: 10.1038/s41378-019-0064-3.

[15] Lake, J.R., Heyde, K.C., Ruder, W.C. (2017). Low-cost feedback-controlled syringe pressure pumps for microfluidics applications. PLOS ONE, 12(4): e0175089. doi: 10.1371/journal.pone.0175089.

[16] Guelig, D., Bauer, J., Wollen, A., Schiller, C., Sherman-Konkle, J., Roche, A., et al. (2017). Design of a Novel, Adjustable Flow Rate, Reusable, Electricity-Free, Low-Cost Syringe Infusion Pump. Journal of Medical Devices, 11(4): 1-6. doi: 10.1115/1.4037935.

[17] Amarante, L.M., Newport, J., Mitchell, M., Wilson, J., Laubach, M. (2019). An Open Source Syringe Pump Controller for Fluid Delivery of Multiple Volumes. Eneuro, 6(5): ENEURO.0240-19.2019. doi: 10.1523/ENEURO.0240-19.2019.

[18] Booeshaghi, A.S., Beltrame, E. da V., Bannon, D., Gehring, J., Pachter, L. (2019). Principles of open source bioinstrumentation applied to the poseidon syringe pump system. Scientific Reports, 9(1): 12385. doi: 10.1038/s41598-019-48815-9.

[19] Pusch, K., Hinton, T.J., Feinberg, A.W. (2018). Large volume syringe pump extruder for desktop 3D printers. HardwareX, 3(November 2017): 49-61. doi: 10.1016/j.ohx.2018.02.001.

[20] Cubberley, M.S., Hess, W.A. (2017). An Inexpensive Programmable Dual-Syringe Pump for the Chemistry Laboratory. Journal of Chemical Education, 94(1): 72-4. doi: 10.1021/acs.jchemed.6b00598.

[21] Garcia, V.E., Liu, J., DeRisi, J.L. (2018). Low-cost touchscreen driven programmable dual syringe pump for life science applications. HardwareX, 4: e00027. doi: 10.1016/j.ohx.2018.e00027. 